ThE ASTROPHYSICAL JouRnAL, 466:352-358, 1996 July 20

(C) 1996. The American Astronomical Society. All rights reserved. Printed in U.S.A.

\title{
ROSAT X-RAY OBSERVATIONS OF FOUR PLANETARY NEBULAE
}

\author{
D. A. LEahy, C. Y. Zhang, K. VolK, and Sun KWoK \\ Department of Physics and Astronomy, University of Calgary, Calgary, Alberta, Canada T2N 1N4 \\ Received 1995 August 9; accepted 1996 February 12
}

\begin{abstract}
We report ROSAT Position Sensitive Proportional Counter (PSPC) observations of four planetary nebulae. The observations show that all four nebulae have two-component spectra. A two-component model consisting of a blackbody and a Raymond-Smith thermal plasma is fitted to the observed ROSAT PSPC spectra of NGC 1535, Abell 36, and NGC 7293. For all three cases, the temperatures of the blackbody component are apparently higher than those of the stellar photospheres, indicating nonblackbody atmospheres. In all cases, the temperatures of the high-energy component can be attributed to a hot plasma associated with the possible stellar coronae or the "hot bubbles" predicted by the interacting winds model. The spectral energy distribution of NGC 1535 is constructed and modeled by incorporating our X-ray spectrum into existing data in UV, optical, near-infrared, and far-infrared wavelengths.

Subject headings: planetary nebulae: individual (NGC 1535, A36, NGC 7293, NGC 3587) X-rays: ISM
\end{abstract}

\section{INTRODUCTION}

A number of planetary nebulae have been detected as $\mathrm{X}$-ray sources. There are three generally accepted possible mechanisms for the production of these X-rays in these sources:

1. The central stars of some planetary nebulae are hot enough to produce soft X-rays from the photosphere;

2. These stars might have coronal regions producing higher energy X-rays; and

3. Some of these stars have high-speed stellar winds that are energetic enough to produce $\mathrm{X}$-rays if they interact with surrounding material.

In the interacting winds model of planetary nebula formation, the high-speed wind from the central star plays a pivotal role in shaping the nebular region, and this wind is shocked because it is confined by previously ejected, lower speed gas. The shock itself might be a source of X-rays. In the simple energy-conserving picture of the interaction of the stellar winds, the planetary nebula develops a hightemperature inner region of gas from the high-speed wind, and the thermal pressure from this gas is responsible for driving the expansion of the planetary nebula (Volk \& Kwok 1985). In such a case, the planetary nebulae will all have high-temperature regions occupying a significant fraction of the total volume of the nebula, which would be an extended X-ray source. The photospheric and possible coronal X-ray emission would occur on much smaller spatial scales.

ROSAT observations of X-rays from $\mathrm{O}$ star winds have recently been analyzed (Hillier et al. 1993). The analysis shows the consistency of the data with two-temperature emission from distributed shocks in an absorbing wind that includes a $\mathrm{He}$ recombination zone. The colliding winds model for the planetary nebula X-ray emission analyzed here is quite different in nature: the shock is at the termination of the wind rather than distributed in the wind (so that the absorption is mainly interstellar rather than internal); the mass-loss rates and X-ray luminosities are much lower than for O stars.

Planetary nebulae are not very strong X-ray sources, and the search for extended emission has not been easy. Some of the earlier reported detections of extended emission (Kreysing et al. 1992) have turned out to be spurious (Chu, Kwitter, \& Kaler 1993). The only confirmed cases of diffuse X-ray emission are NGC 6543 (Kreysing et al. 1992) and Abell 30 (Chu \& Ho 1995). Recently, Leahy, Zhang, \& Kwok (1994, hereafter Paper I) have suggested that the "hot bubble" predicted by the interacting winds model could be identified by spectral analysis. The planetary nebula NGC 7293 was found to show a two-component $\mathrm{X}$-ray spectrum, where one component is roughly consistent with the expected central star photospheric emission, and the second component is consistent with emission from a high-temperature, optically thin plasma similar to the expected "hot bubble."

In this paper, we apply the same technique used for NGC 7293 and discuss new ROSAT observations of three planetary nebulae (including NGC 7293), all of which show strong evidence of having two-component X-ray spectra. The fourth planetary nebula has been detected by ROSAT, although no spectrum could be extracted.

\section{NGC 1535}

NGC 1535 was observed for a total of $11,156 \mathrm{~s}$ with the ROSAT Position Sensitive Proportional Counter (PSPC) instrument. During this period, about 500 photons were detected within $10^{\prime}$ of the nominal position of the nebula, some of which are due to the X-ray background. The X-ray spectrum was extracted using the XRAY package under the IRAF data reduction software. After background subtraction, 268 photons were detected within a $6^{\prime}$ radius of the source position as determined from the ROSAT observation: $\alpha(2000.0)=04^{\mathrm{h}} 14^{\mathrm{m}} 17^{\mathrm{s}} .6, \delta(2000.0)=-12^{\circ} 43^{\prime} 19^{\prime \prime} .7$. This position is consistent with the optical position within the ROSAT pointing accuracy. For this observation, a significant amount of low-energy X-ray background was present.

The spectrum that was found has a "double-hump" shape, with approximately one-third of the photons in the high-energy $(>0.5 \mathrm{keV})$ range, and the remaining photons peaking at about $0.25 \mathrm{keV}$. The extended range of energies for the photons shows that the emission is not simply due to the stellar photosphere, for even a $250,000 \mathrm{~K}$ central star 
would not produce $X$-rays with an energy of $1 \mathrm{keV}$ (corresponding temperature $\approx 1.2 \times 10^{7} \mathrm{~K}$ ). The spectrum was fitted using the XSPEC X-ray spectrum analysis package. The types of models chosen were either blackbodies or optically thin plasma models, the latter using the Raymond-Smith plasma code, subject to some interstellar extinction.

The amount of extinction for this nebula is not well known. The $\mathrm{H} \alpha / \mathrm{H} \beta$ emission-line strength observed for NGC 1535 is consistent with the results of the case B recombination theory, implying that there is little or no extinction for this nebula. Comparison of the $\mathrm{H} \beta$ line flux with the 5 $\mathrm{GHz}$ radio flux density gives another extinction estimate. Acker et al. (1992) gives 15 and $5 \mathrm{GHz}$ radio flux densities of 0.171 and $0.141 \mathrm{Jy}$ for this nebula. Correcting the $15 \mathrm{GHz}$ flux density value to $5 \mathrm{GHz}$, assuming the spectrum to be due to thermal bremsstrahlung, leads to $C(\mathrm{H} \beta)$ estimates of 0.088 and 0.052 , respectively. Taking a mean value of $C(\mathrm{H} \beta)=0.07+0.03$ and carrying out the standard conversion to $E(B-V)$ as in Seaton (1979) gives a value of $0.046 \pm 0.020 \mathrm{mag}$, which corresponds to $N_{\mathrm{H}}=$ $\left(2.8 \pm 1.2 \times 10^{20}\right) \mathrm{cm}^{-2}$. The nominal value was set as the extinction to NGC 1535 .

Fits to the observed spectrum were carried out for three possible models. First, fits were sought with blackbody models and standard Raymond-Smith plasma models. Each of these models have two parameters. With 34 data bins to fit, the number of degrees of freedom is 32 . The best-fit $\chi^{2}$ values were found to be 40.4 and 47.1 for these two types of plasma models. Expressed in terms of $\chi^{2} / v$, where $v$ denotes the number of degrees of freedom, the fits have reduced $\chi^{2}$ values of 1.26 and 1.47 , respectively.

A significantly better fit is obtained for a nodel including both a blackbody component and a plasma model component. The results of this fit are shown in Figure 1: the top panel shows the sum of the two components (the data are given by the crosses); the middle panel shows the contribution of the blackbody component by the histogram; and the bottom panel shows the contribution of the plasma component. Using the two-component model, $v$ becomes 30 and the best-fit $\chi^{2}$ is reduced to 25.8 , giving a $\chi^{2} / v$ value of 0.86 . Thus, the fit is acceptable within the uncertainties of the data values. The best-fit parameters were found to be a blackbody temperature of $356,000 \mathrm{~K}(k T=0.0307 \mathrm{keV})$, a blackbody normalization of $8.5 \times 10^{-6}\left(L_{*} / d^{2}\right.$ in units of $10^{37} \mathrm{ergs} \mathrm{s}^{-1} \mathrm{kpc}^{-2}$ ), a plasma temperature of $5.7 \times 10^{6} \mathrm{~K}$ $(k T=0.492 \mathrm{keV})$, and a plasma component normalization of $2.27 \times 10^{-5}$ (emission measure divided by the distance squared in units of $10^{-14} \mathrm{~cm}^{-5}$. The $90 \%$ confidence intervals in the temperatures are $(0.031-1.0) \times 10^{6} \mathrm{~K}$ for the blackbody component and (3.1-8.9) $\times 10^{6} \mathrm{~K}$ for the plasma component.

The blackbody component has a large temperature uncertainty but is consistent with emission from the central star, which has an estimated temperature of $70,000 \mathrm{~K}$ (Mendez et al. 1988; see also $\S 6.3$ below). The hot plasma component can be identified with either a corona or a shocked stellar wind region in the nebula. The normalization of the blackbody corresponds to a radius of about $50 \pm 6 \mathrm{~km}$ for the central star, if the distance of the nebula is in a range of 1.5 (Zhang 1993) to $2.1 \mathrm{kpc}$ (Mendez et al. 1988). The estimated blackbody radius of the central star is very small compared with the expected radius of a white dwarf star. This is likely to be a reflection of the non-

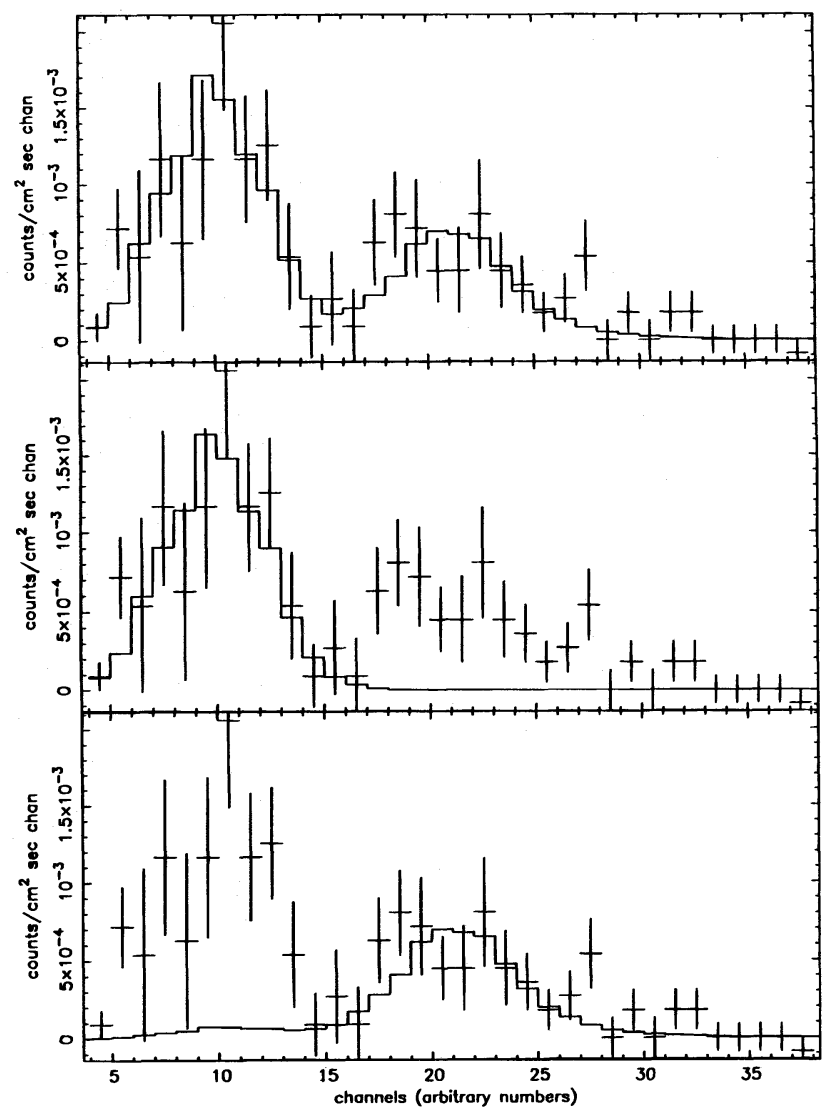

Fig. 1.-ROSAT PSPC spectrum of NGC 1535. The data are plotted in the top panel as crosses (with vertical bars showing the $\pm \sigma$ uncertainties). The model is the blackbody plus plasma model of Table 1. The lower panels show separately the contributions to the model of the blackbody component (middle panel) and of the plasma component (bottom panel). See Table 2 for conversion of channel number to energy.

blackbody nature of the actual stellar spectrum, although in principle it could indicate that discrete hot spots on the stellar surface are the source of the X-ray emission.

There is no evidence, at the angular resolution of the PSPC instrument, that NGC 1535 has any extended X-ray emission. The radial profiles for the X-rays in both the lowenergy and the high-energy ranges are consistent with the ROSAT point-spread function. In particular, the higher energy photons, which the model fit suggests come from the plasma component, do not show any evidence of being extended on scales of more than $30^{\prime \prime}$. The observations of NGC 1535 were carried out during eight blocks of time. The source count rate varied by a factor of about 2 between these different individual observations. One observation of length $1884 \mathrm{~s}$ detected $40 \%$ of the total number of photons, although it represents $17 \%$ of the total time of observation. The cause of this fluctuation is unknown. (Instrumental effects cannot be ruled out.) Within any individual time interval, there is no evidence of any periodic effects in the data values, as determined from fast Fourier transform (FFT) analysis of each of the eight blocks of time.

\section{ABELL 36}

The planetary nebula A36 was observed twice with the ROSAT PSPC instrument, first in the normal mode, for an integration time of $9990 \mathrm{~s}$, and then with a boron filter in place, for $9600 \mathrm{~s}$. The boron filter screens out most of the 
low-energy photons and changes the energy response to the high-energy photons, thereby aiding in modeling the source spectrum. A36 was detected in both observations, at a somewhat lower count rate than was NGC 1535.

The source position determined from the ROSAT observations was $\quad \alpha(2000.0)=13^{\mathrm{h}} 40^{\mathrm{m}} 21^{\mathrm{s}} .7, \quad \delta(2000.0)=$ $-19^{\circ} 51^{\prime} 48^{\prime \prime}$. 2 . The background-corrected total counts were 107 and 49 within $6^{\prime}$ of the source position for the normal and boron-filter observations, respectively. Since the total number of counts is low, only the spectral analysis was carried out for this source. The fitting was done in XSPEC as for NGC 1535, but now both data sets were fitted simultaneously. The gap in the spectrum from the no-filter observation of A36 (left side of Fig. 2) is due to omission of four data channels spanning $0.84-1.15 \mathrm{keV}$, where the data values oscillated between large count values and negative net count values. The cause of these anomalous data values is unknown. While an individual channel might be subject to a statistical fluctuation, producing either an anomalously large or small value, it is unlikely that four consecutive channels would exhibit large fluctuations by chance alone. It was therefore judged best to remove these channels from consideration. However, those same channels in the boronfilter observation showed no anomaly and were included in the spectral fitting (right side of Fig. 2).

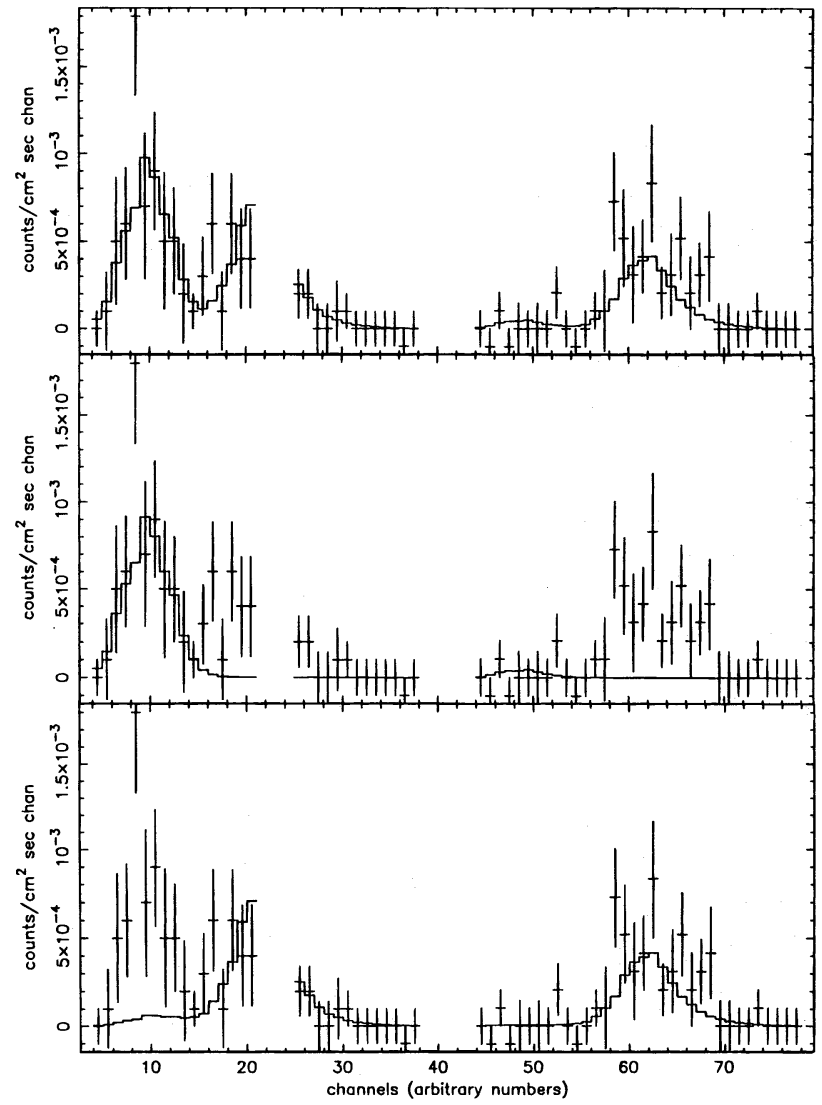

FIG. 2.-ROSAT PSPC spectrum of Abell 36. The data are plotted in the top panel as crosses (with vertical bars showing the $\pm \sigma$ uncertainties). The PSPC observation without filter is located on channels 4-37; the PSPC observation with boron filter is located on channels 44-77. The model is the blackbody plus plasma model of Table 1 . The lower panels show separately the contributions to the model of the blackbody component (middle panel) and of the plasma component (bottom panel). See Table 2 for conversion of channel number to energy.
The extracted spectrum showed the same type of "double-hump" shape as was seen in NGC 1535, but in this case the higher energy photons represent more than $50 \%$ of the total counts. The fitting was carried out in the same general way as for NGC 1535. For A36, the extinction value derived from comparison of the $\mathrm{H} \beta$ flux and the $5 \mathrm{GHz}$ flux density is absurdly large, $C(\mathrm{H} \beta)=0.41$, which probably means that the radio flux density was not measured accurately. Thus, for this nebula, an $E(B-V)$ value derived from the optical Balmer line ratios was taken from the literature (Kaler \& Feibelman 1985), and the corresponding $N_{\mathrm{H}}$ of $2.9 \times 10^{20} \mathrm{~cm}^{-2}$ was used for all of the fits.

The one-component $\chi^{2}$ values for the blackbody model and the plasma model were 51.6 and 59.5, respectively, for 64 data bins and two parameters. While these fits are formally acceptable, they both systematically fail at low energies. The main reason that the $\chi^{2} / v$ values are less than 1 is that the average number of photons per bin is of order 2 , and so the poor counting statistics allow leeway for fitting. When a two-component model was used, a $\chi^{2}$ value of 37.4 for the 64 data bins and four parameters was obtained. The $\chi^{2} / v$ value is 0.62 for this model. It fits the data much better than either one-component model does. The results of the two-component fit for Abell 36 are shown in Figure 2: the top panel shows the sum of the two components (the data are given by the crosses, with the boron-filter data on the right and the no-filter data on the left); the middle panel shows the contribution of the blackbody component by the histogram; and the bottom panel shows the contribution of the plasma component.

The temperatures derived for the two components are $3.0 \times 10^{6} \mathrm{~K}(k T=0.0259 \mathrm{keV})$ for the blackbody component and $7.1 \times 10^{6} \mathrm{~K}(k T=0.612 \mathrm{keV})$ for the plasma component, values that are very similar to those for NGC 1535. The normalizations, in the same units as above, are $10^{-4}$ for the blackbody component and $2.26 \times 10^{-5}$ for the plasma component. The $90 \%$ confidence intervals in the temperatures are $(0.181-0.810) \times 10^{6} \mathrm{~K}$ for the blackbody component and (4.2-9.9) $\times 10^{6} \mathrm{~K}$ for the plasma component. The temperature derived for the blackbody component is probably somewhat higher than the actual stellar temperature, which is unlikely to be greater than $0.15 \times 10^{6}$ $\mathrm{K}$. It is noted that the hydrogen Zanstra temperature of the central star of A36 is $0.073 \times 10^{6} \mathrm{~K}$ (Kaler \& Feibelman 1985); in addition, the optical spectrum is assigned spectral type 07 , which argues against the temperature being extremely high. As for NGC 1535, the low value of the normalization constant for the blackbody component probably reflects the nonblackbody nature of the stellar spectrum at these wavelengths.

\section{NGC 7293}

The ROSAT spectrum of NGC 7293, the Helix Nebula, has previously been discussed in Paper I. The data used in this paper comes from the normal observation mode of ROSAT. An additional observation of NGC 7293 using the boron filter was also made, and we have reduced these data and carried out fitting for both sets of data.

The X-ray emission from NGC 7293 is similar in general nature to that of NGC 1535 and A36, but with a comparatively weaker hard X-ray component. The results of the two-component fit for NGC 7293 are shown in Figure 3: the top panel shows the sum of the two components (the data are given by the crosses, with the boron-filter data on 


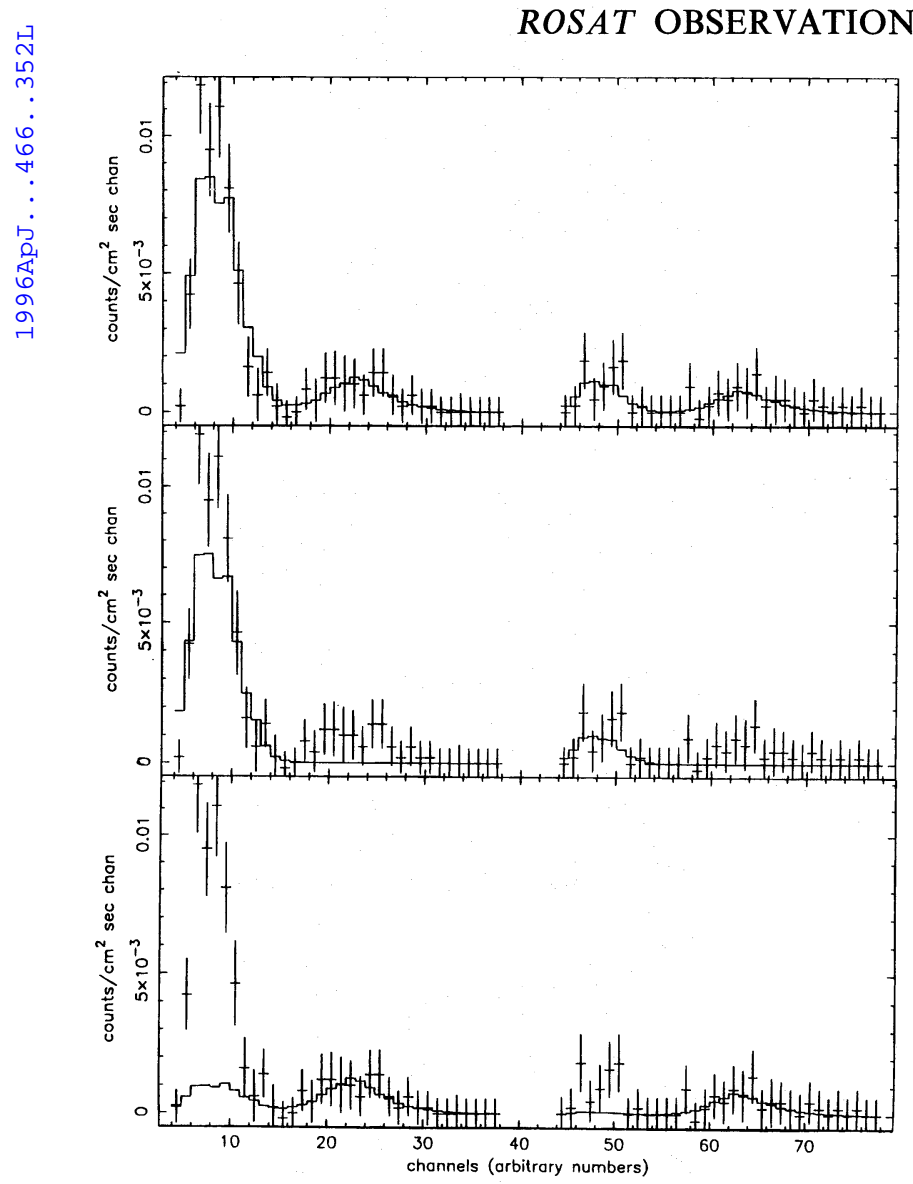

Fig. 3.-ROSAT PSPC spectrum of NGC 7293. The data are plotted in the top panel as crosses (with vertical bars showing the $\pm \sigma$ uncertainties). The PSPC observation without filter is located on channels 4-37; the PSPC observation with boron filter is located on channels 44-77. The model is the blackbody plus plasma model of Table 1 . The lower panels show separately the contributions to the model of the blackbody component (middle panel) and of the plasma component (bottom panel). See Table 2 for conversion of channel number to energy.

the right and the no-filter data on the left); the middle panel shows the contribution of the blackbody component by the histogram; and the bottom panel shows the contribution of the plasma component.

TABLE 1

SPECTRAL PARAMETERs ${ }^{\mathrm{a}}$

\begin{tabular}{llll}
\hline \hline \multicolumn{1}{c}{ Parameter } & NGC 7293 & NGC 1535 & \multicolumn{1}{c}{ A36 } \\
\hline$N_{\mathrm{H}}\left(10^{20} \mathrm{~cm}^{-2}\right)^{\mathrm{b}} \ldots \ldots \ldots \ldots$. & 1.9 & 2.8 & 2.9 \\
Blackbody $k T(\mathrm{keV}) \ldots \ldots \ldots$ & $0.023_{-0.005}^{+0.006}$ & $0.031_{-0.028}^{+0.058}$ & $0.026_{-0.010}^{+0.044}$ \\
Normalization $\mathrm{c} \ldots \ldots \ldots \ldots \ldots$ & $5.7_{-2.4}^{+5.9}$ & $8.5_{-2.5}^{+340}$ & $10.0_{-8.8}^{+230}$ \\
Plasma $k T(\mathrm{keV}) \ldots \ldots \ldots \ldots$ & $0.93_{-0.29}^{+0.36}$ & $0.49_{-0.22}^{+0.41}$ & $0.61_{-0.25}^{+0.24}$ \\
Normalization $\left(10^{-5}\right)^{\mathrm{d}} \ldots \ldots$. & $6.3_{-4.0}^{+19}$ & $2.3_{-0.7}^{+2.3}$ & $2.3_{-0.6}^{+0.7}$ \\
\hline
\end{tabular}

${ }^{\text {a }}$ The best-fit parameters for the ROSAT X-ray spectra of the three planetary nebulae. Each spectrum has been fitted with a blackbody component and a Raymond-Smith plasma component. The best-fit parameters and their $90 \%$ confidence ranges are given for the four variable parameters.

b The interstellar column $\left(N_{H}\right)$ values are adopted from estimates of the extinction $E(B-V)$ based on values given in the literature. In all three cases, additional fitting with $N_{\mathrm{H}}$ as a free parameter established that the range of possible $N_{\mathrm{H}}$ values estimated from the X-ray spectra was consistent with the extinction values available for these nebulae.

c The normalization values for the blackbody components are $L_{*} / d^{2}$ in units of $10^{37} \mathrm{ergs} \mathrm{s}^{-1} \mathrm{kpc}^{-2}$, where $L_{*}$ is the total source luminosity and $d$ is the source distance.

${ }^{d}$ For the plasma component, the normalization values are the plasma emission measures divided by the distance squared, in units of $10^{-14} \mathrm{~cm}^{-5}$.

The best-fit model for the spectrum has a lower temperature blackbody component and a higher temperature Raymond-Smith plasma component. The ROSAT observation with the boron filter produces relatively few counts for NGC 7293 because it eliminates a large fraction of the photons from the blackbody component. The relatively small number of counts per channel for the boron observation produces rather large relative uncertainties, so the second spectrum does not produce nearly as strong constraints on a model fit as does the normal ROSAT observation. This makes the $\chi^{2} / v$ value lower than it should be. The best-fit blackbody plus plasma model produces a $\chi^{2}$ of 32.96 for 68 data bins and four parameters, for a $\chi^{2} / v$ value of 0.51 . The value would probably increase by about $50 \%$ if only those channels with several counts were used from the boron-filter data.

The blackbody component parameters are a temperature of $0.27 \times 10^{6} \mathrm{~K}(\mathrm{kT}=0.0234 \mathrm{keV})$ and a normalization of $5.66 \times 10^{-2}$, while the plasma component parameters are a temperature of $1.08 \times 10^{7} \mathrm{~K}(k T=0.930 \mathrm{keV})$ and a normalization of $6.28 \times 10^{-5}$. The best-fit temperatures are both somewhat higher than is found for the best fit to the unfiltered observation alone, but the effect is significant only for the plasma component that is more constrained by the boron-filter observation. These values and the best-fit values for NGC 1535 and A36 are given in Table 1. (We note that the main contributing factor to the higher temperature for the blackbody component obtained here compared with Paper I is the use of the updated PSPC response matrix.) Table 2 shows the conversion of channel number to energy.

\section{NGC 3587}

This planetary nebula was observed by ROSAT for 1783 $\mathrm{s}$ in the normal mode and for $4147 \mathrm{~s}$ in the boron-filter mode. Only a marginal detection resulted in both observations. The estimated numbers of counts near the source position on the images after background subtraction were $8.6 \pm 4.2$ counts for the unfiltered observation and $6.9 \pm 3.0$ counts for the boron-filter observation. The uncertainties given are $1 \sigma$ values and mostly reflect the counting statistics. The detections are at about 10 times the background count rate. The resulting count rates are $(4.8 \pm 2.4) \times 10^{-3}$ $\mathrm{s}^{-1}$ for the unfiltered observation and $(1.7 \pm 0.8) \times 10^{-3}$ $\mathrm{s}^{-1}$ for the boron-filter observation.

The unfiltered observation appears to have detected the source primarily in the lower energy channels, while the boron-filter observation detected higher energy photons. Assuming that the two observations detected photons in separate energy bands, a hardness ratio can be calculated for the spectrum of NGC 3587. Using a ratio of the form (high-energy count rate minus low-energy count rate)/(total count rate), one obtains a value of -0.5 , with a rather large uncertainty. This hardness ratio should roughly correspond to one of the hardness ratio estimates provided in the ROSAT standard data reduction. The values for NGC 7293 and NGC 1535 are $-0.56 \pm 0.05$ and $-0.05 \pm 0.11$, respectively. The hardness ratio for NGC 3587 appears to be like that of NGC 7293 rather than that of NGC 1535, implying a somewhat weaker high-temperature component.

\section{SPECTRAL ENERGY DISTRIBUTION OF NGC 1535}

A systematic investigation of spectral energy distributions of 66 compact planetary nebulae over a wide wave- 
length coverage from UV to far-infrared by Zhang \& Kwok (1991) has led to a detailed breakdown of the energy budget of those objects. It is found that, on the average, $38 \%, 25 \%$, and $37 \%$ of the emergent fluxes in the above wavelength range are emitted, respectively, by the central star, gaseous nebula, and dust shell. Models of the overall spectral evolution of planetary nebulae with time have been shown to be useful in furthering our understanding of the nature and evolution of these objects (Volk 1992). With our newly obtained ROSAT X-ray spectra, it is now possible for us to extend the wavelength coverage to include the energy emitted in the X-ray.

\subsection{Observed Spectral Energy Distribution}

We will concentrate on the spectral energy distribution of NGC 1535, because of its moderate size of about $20^{\prime \prime}$ in diameter. This ensures that almost all the fluxes emitted by the nebula should have been detected through the large entrance aperture $\left(10^{\prime \prime} \times 20^{\prime \prime}\right)$ of the International Ultraviolet Explorer (IUE) spectroscopy and by broadband photometry at other wavelengths. (We note that the other three planetary nebulae observed here do not have reliably measured absolute fluxes at other wavelengths due to their spatial extension, so that it is not feasible to give their spectral energy distributions.)

In Figure 4, we plot $\lambda F_{\lambda}$ versus $\lambda$ for NGC 1535 over a wavelength range from about $10 \AA$ to $100 \mu \mathrm{m}$. Our observed $\mathrm{X}$-ray spectrum is shown as a thin solid line covering the wavelength range from 10 to $100 \AA$. The IUE lowdispersion images, SWP 10737 and LWR 2232, of NGC 1535 were retrieved and processed at the IUE Data Analysis Center (IUEDAC). The short-wavelength (SWP 10737) and long-wavelength (LWR 2232) parts of the spectrum were then joined at $1975 \AA$ to produce a single, result- ant spectrum. In Figure 4, only the IUE data in the range from 1250 to $3250 \AA$ are presented. The total $U, B$, and $V$ magnitudes of the nebula as a whole were taken from Feinstein (1985). These data are shown as open diamonds in Figure 4. The $B$ and $V$ magnitudes of the central star as determined from the continuum levels of the optical spectrum taken from Tylenda et al. (1991) are plotted as open circles in Figure 4. The near-infrared magnitudes of $J, H$, and $K$ are from Whitelock (1985) and are shown as open squares in Figure 4. The IRAS fluxes at the four wavelength bands of $12,25,60$, and $100 \mu \mathrm{m}$ are indicated by filled circles in Figure 4. All these observed fluxes plotted are uncorrected for interstellar extinction.

\subsection{Model}

We have made a model fit to the spectral energy distribution from 0.1 to $100 \mu \mathrm{m}$, using a procedure described in Zhang \& Kwok (1991). The model consists of three components, i.e., the stellar photospheric continuum flux, the nebular gaseous continuum flux, and the dust thermal emission flux. The stellar continuum is approximated by a blackbody at a color temperature $T_{*}$. The nebular continuum is composed of the free-free and free-bound emission of $\mathrm{H} \mathrm{I}, \mathrm{He} \mathrm{I}$, and $\mathrm{He} \mathrm{II}$, and the hydrogen two-photon emission. The nebular emissivity depends on the helium abundances $\left(\mathrm{He}^{+} / \mathrm{H}\right.$ and $\left.\mathrm{He}^{++} / \mathrm{H}\right)$, electron density $\left(N_{e}\right)$, and electron temperature $\left(T_{e}\right)$. The dust emission is represented as a blackbody at a dust temperature of $T_{d}$.

Since we are interested in the continuum, we have binned the IUE data to measure the continuum in that wavelength range, with a constant bin width of $60 \AA$, avoiding any absorption and emission lines. When we apply the model to the data, we have fitted to fluxes corrected for the extinction of $E(B-V)=0.046$ (see $\S 2$ above). Taking $N_{e}=4200$

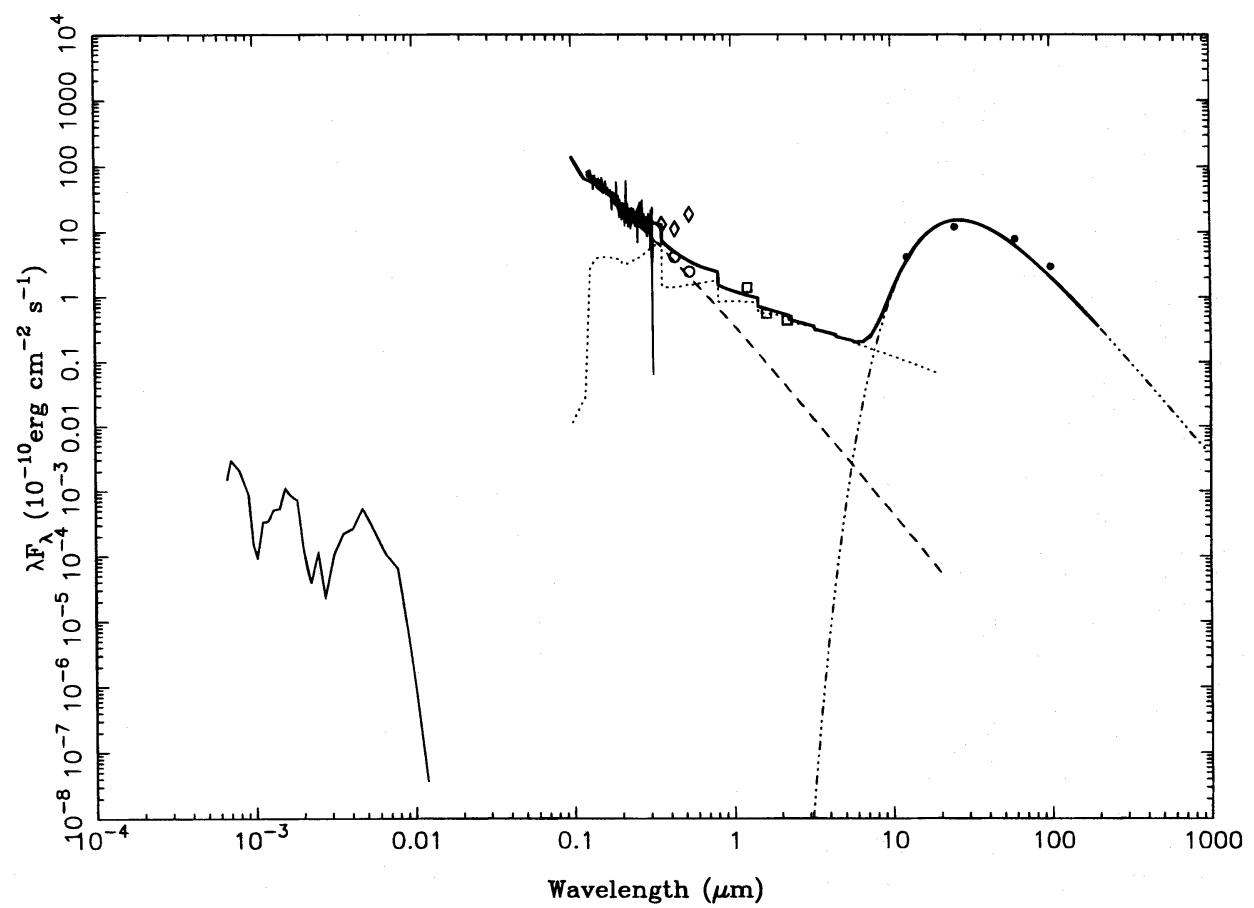

Fig. 4.- Spectral energy distribution of NGC 1535 in the wavelength range from $10 \AA$ to $100 \mu \mathrm{m}$. Thin line curves are the ROSAT X-ray and IUE spectra. Dots are $I R A S$ survey measurements. Open squares are near-infrared $J H K$ measurements. Open diamonds are total $U, B$, and $V$ magnitudes of the nebula as a whole. Open circles are $B$ and $V$ magnitudes of the central star. Model curves for the stellar, nebular, and dust components are shown as dashed, dotted, and dash-dotted lines, respectively. The thick solid line shows the synthesized spectral energy distribution, which is the sum of the above three components. The observed fluxes are not corrected for extinction, while the model fluxes are reddened. 
$\mathrm{cm}^{-3}, \quad T_{e}=11,000 \mathrm{~K}, \mathrm{He}^{+} / \mathrm{H}=0.081, \quad$ and $\mathrm{He}^{++} /$ $\mathrm{H}=0.013$ (Pottasch 1984), we have calculated the nebular continuum emission. After that we have subtracted the model nebular continuum fluxes from the observed, dereddened fluxes at each wavelength. We have then fitted the stellar and dust parts of the model to the difference continuum spectrum. The resultant model fluxes are reddened in order to be compared with the observed data.

In Figure 4, we show the three components of the model, along with the summation of the three. It is clear that our model can fit the observed continuum spectrum from ultraviolet through far-infrared. It is noted that the $B$ and $V$ magnitudes for the nebula as a whole by Feinstein (1985) exceed the model predicted fluxes, while the $U$-band magnitude of Feinstein (1985) is in agreement with the model and with extrapolation from the IUE spectra. The discrepancy may be caused by the fact that the aperture size of $22^{\prime \prime}$ used by Feinstein (1985) is larger than that used for the IUE observations. It is, however, more important to realize that the broadband measurements of Feinstein (1985) contain not only the contribution from the continuum but also that from the strong emission lines in the optical, which are not shown in Figure 4. For example, the [O III] $\lambda 5007$ line dominates the flux seen in the broad $V$ band. On the other hand, the $B$ and $V$ magnitudes of the central star are fairly well represented by the blackbody curve for the central star in our model, since these stellar fluxes were obtained by carefully subtracting the contribution from the nebular emission in the spectrum (Tylenda et al. 1991).

\subsection{Results}

From the model fitting, we obtain some important physical parameters for NGC 1535 . The color temperature of the central star is in good agreement with that of $70,000 \mathrm{~K}$ determined from the analysis of the stellar absorption line profiles by Mendez et al. (1988). The angular radius of the central star is found to be about $1^{\prime \prime} .03 \times 10^{-6}$ arcsec. For the dust shell, we obtain the dust temperature of $137 \mathrm{~K}$ and the optical depth of $3.8 \times 10^{-5}$ at $100 \mu \mathrm{m}$.

It is most interesting that the model fitting results in a detailed breakdown of the energy budget. The flux $\left(F_{*}\right)$ of the nonionizing radiation of the central star, the flux $\left(F_{n}\right)$ of the nebular gaseous continuum emission, and the farinfrared flux are obtained, respectively, as 140, 18.9, and 43.9 times the dereddened $\mathrm{H} \beta$ flux $\left(F_{\mathrm{H} \beta}\right)$, which is $4.65 \times 10^{-11}$ ergs cm ${ }^{-2} \mathrm{~s}^{-1}$. The total flux $\left(F_{l}\right)$ from emission lines cannot be obtained from the model fitting to the continuum. We can, however, estimate $F_{l}$ as follows. In deriving the energy balance temperature of the central star of NGC 1535, Preite-Martinez \& Pottasch (1983) have estimated the ratio of the total flux of the "collisionally excited" lines to the $\mathrm{H} \beta$ line as 22.4. The total flux from the $\mathrm{H}$ I recombination lines of $\mathrm{H} \alpha, \mathrm{H} \beta, \mathrm{H} \gamma$, and $\mathrm{H} \delta$ for case $\mathrm{B}$ is about $4.8 F_{\mathrm{H} \beta}$ from the case $\mathrm{B}$ recombination line calculations (Brocklehurst 1971). Similarly, the total flux of He II lines 1640,2734 , and $4696 \AA$ is about 7.8 times that of the He II $\lambda 4868$ line (Brocklehurst 1972). The He II $\lambda 4868$ flux corrected for extinction is measured as $0.18 F_{\mathrm{H} \beta}$ by Aller \& Czyzak (1978). Therefore, the flux from the He II lines amounts to $1.3 F_{\mathbf{H} \beta}$. Finally, we estimate that the total flux from recombination and collisionally excited lines is $F_{l}=$ $29 F_{\mathrm{H} \beta}$. The model fit to the ROSAT X-ray spectrum gives $\mathrm{X}$-ray fluxes of $4.1584 \times 10^{-13}$ and $6.0348 \times 10^{-14} \mathrm{ergs}$ $\mathrm{cm}^{-2} \mathrm{~s}^{-1}$, respectively, in the blackbody and plasma com- ponents. The results translate to the flux of the blackbody component being $0.0089 F_{\mathrm{H} \beta}$ and that of the plasma component being $0.0013 F_{\mathrm{H} \beta}$.

In summary, the total dereddened flux observed from the $\mathrm{X}$-ray through the far-infrared amounts to $232 F_{\mathrm{H} \beta}$, while the flux in nonionizing radiation of the central star, the flux in the nebular gaseous continuum and emission lines, and the flux from the dust shell are $60 \%, 19 \%$, and $21 \%$, respectively, of the total observed flux. The contribution from both the soft and hard X-ray components is negligible in comparison with the other components.

\section{DISCUSSION AND CONCLUSIONS}

Three planetary nebulae have been found to have twocomponent X-ray spectra, which can be interpreted as arising from the photosphere and the shocked stellar wind, respectively. The cooler component is interpreted as arising from the photosphere, although, in all three cases, the blackbody temperature (Table 1) is higher than the effective temperature of the central star, as determined by other means than X-ray data. This is likely due to the nonthermal nature of the photospheric spectrum. This has been suggested in models for central star atmospheres (e.g., Gabler, Kudritzki, \& Mendez 1991) and is also consistent with the low value of the blackbody-emitting area found here.

TABLE 2

CHANNEL-TO-ENERGY CONVERSION ${ }^{\mathrm{a}}$

\begin{tabular}{|c|c|c|}
\hline Channel Numbers & $E_{\text {low }}$ & $E_{\text {high }}$ \\
\hline 4,44 & 0.07 & 0.09 \\
\hline 5,45 & 0.09 & 0.11 \\
\hline 6,46 & 0.11 & 0.14 \\
\hline 7,47 . & 0.14 & 0.17 \\
\hline 8,48 & 0.17 & 0.20 \\
\hline 9,49 & 0.20 & 0.24 \\
\hline $10,50$. & 0.24 & 0.28 \\
\hline $11,51 \ldots$ & 0.28 & 0.32 \\
\hline $12,52 \ldots$ & 0.32 & 0.37 \\
\hline $13,53 \ldots$ & 0.37 & 0.42 \\
\hline 14,54 . & 0.42 & 0.47 \\
\hline $15,55$. & 0.47 & 0.52 \\
\hline $16,56 \ldots$ & 0.52 & 0.58 \\
\hline $17,57 \ldots$ & 0.58 & 0.64 \\
\hline $18,58$. & 0.64 & 0.70 \\
\hline 19,59 . & 0.70 & 0.77 \\
\hline 20,60 . & 0.77 & 0.84 \\
\hline $21,61 \ldots$ & 0.84 & 0.91 \\
\hline $22,61 \ldots$ & 0.91 & 0.99 \\
\hline $23,63 \ldots$ & 0.99 & 1.07 \\
\hline $24,64 \ldots$ & 1.07 & 1.15 \\
\hline $25,65 \ldots$ & 1.15 & 1.23 \\
\hline 26,66 . & 1.23 & 1.32 \\
\hline 27,67 . & 1.32 & 1.41 \\
\hline $28,68 \ldots$ & 1.41 & 1.50 \\
\hline $29,69 \ldots$ & 1.50 & 1.60 \\
\hline $30,70 \ldots$ & 1.60 & 1.70 \\
\hline $31,71 \ldots$ & 1.70 & 1.80 \\
\hline 32,72 . & 1.80 & 1.91 \\
\hline 33,73 . & 1.91 & 2.02 \\
\hline $34,74 \ldots$ & 2.02 & 2.13 \\
\hline $35,75 \ldots$ & 2.13 & 2.24 \\
\hline 36,76 . & 2.24 & 2.36 \\
\hline $37,77 \ldots$ & 2.36 & 2.48 \\
\hline
\end{tabular}

a The lower and upper energy boundaries (in $\mathrm{keV}$ ) for each channel number are given (for use with Figs. 1-3). The channels 4 and 44, 5 and 45, 6 and $46, \ldots, 37$ and 77 have identical energy boundaries. 
We note that the temperature of the hot component is significantly higher than the value found for the diffuse component of Abell 30 (Chu \& Ho 1995). Assuming that the "hot bubble" fills the entire inner region of the nebula, equation (3) of Paper I gives a temperature of $0.43(v / 1000$ $\left.\mathrm{km} \mathrm{s}^{-1}\right)^{2} \mathrm{keV}$, where $v$ is the velocity of the central star wind. For typical observed central star wind velocity of 2000-4000 $\mathrm{km} \mathrm{s}^{-1}$ (Cerruti-Sola \& Perinotto 1985), our observed temperatures are very similar to the predicted values.

It is possible that the temperature of the "hot bubble" decreases with age as the result of heat loss by conduction, and an older nebula like Abell 30 could have much lower bubble temperatures. Unfortunately, the emission measure of the bubble (and therefore the X-ray flux) is also expected to decrease with time (Volk \& Kwok 1985), and, therefore, the chance of detecting diffuse emission from evolved planetary nebulae also becomes much less unless the nebula is nearby. The case of Abell 30 is further complicated by the fact that it is likely a "born-again " planetary nebula, based on its peculiar chemical composition (Iben 1993). Its low $\mathrm{X}$-ray temperature could be the result of the new stellar wind having to fill a larger volume vacated by the old expanding shell.

While it is now generally believed that all planetary nebulae are created by an interacting winds process, the $\mathrm{P}$ Cygni profiles associated with the fast central wind are detected in less than $50 \%$ of the cases. Since the central stellar wind mass-loss rate is expected to decrease with time and makes direct detection of the wind in the UV difficult, the search for the remnant X-ray hot bubble may represent a better method for finding the evidence of the interacting winds history of evolved nebulae.

Given the limited spatial resolution and sensitivity of present X-ray telescopes, the spectral analysis method may represent a more practical way to detect the shocked stellar winds in planetary nebulae. If the "hot bubbles" of a larger sample of planetary nebulae can be detected using this method, then the temperatures and emission measures derived from the X-ray observations can be used to constrain the dynamical model of planetary nebula evolution.

For the first time, the spectral energy distribution of NGC 1535 has been constructed by combining our recent ROSAT data with the existing UV, optical, and infrared data. The detailed breakdown of the energy budget resulting from the modeling of the spectral energy distribution has shown that, while $60 \%$ of the observed flux comes from the nonionizing radiation of the central star, the rest is seen in the nebular gas and dust shell emission. The contribution from the X-ray component is negligible in the entire energy budget.

D. A. L. and S. K. acknowledge support from the Natural Sciences and Engineering Research Council of Canada.

\section{REFERENCES}

Acker, A., Ochsenbein, F., Stenholm, B., Marcout, J., \& Schohn, C. 1992, Strasbourg-ESO Catalogue of Galactic Planetary Nebulae (Garching: ESO)

Aller, L. H., \& Czyzak, S. J. 1978, Ap\&SS, 62,397

Brocklehurst, M. 1971, MNRAS, 153, 471 1972, MNRAS, 157, 211

Cerruti-Sola, M., \& Perinotto, M. 1985, ApJ, 291, 237

Chu, Y.-H., \& Ho, C.-H. 1995, ApJ, 448, L127

Chu, Y.-H., Kwitter, K., \& Kaler, J. 1993, AJ, 106,650

Feinstein, A. 1985, Rev. Mexicana Astron. Astrofiz., 10,203

Gabler, R., Kudritzki, R. \& Mendez, R. 1991, A\&A, 245,587

Hillier, D., Kudritzki, R., Pauldrach, A., Baade, D., Cassinelli, J., Puls, J., \& Schmitt, J. 1993, A\&A, 276, 117

Iben, I. 1993, in IÁU Symp. 155, Planetary Nebulae, ed. R. Weinberger \&

A. Acker (Dordrecht: Kluwer), 587

Kaler, J. B., \& Feibelman, W. A. 1985, ApJ, 297, 724
Kreysing, H. C., Diesch, D., Zweigle, J., Staubert, R., Grewing, M., \& Hasinger, G. 1992, A\&A, 264, 623

Leahy, D. A., Zhang, C. Y., \& Kwok, S. 1994, ApJ, 422, 205 (Paper I)

Mendez, R. H., Kudritzki, R. P., Herrero, A., Husfeld, D., \& Groth, H. G. 1988, A\&A, 190, 113

Pottasch, S. R. 1984, Planetary Nebulae (Dordrecht: Reidel)

Preite-Martinez, A., \& Pottasch, S. R. 1983, A\&A, 126, 31

Seaton, M. J. 1979, MNRAS, 187,73P

Tylenda, R., Acker, A., Stenholm, B., Gleizes, F., \& Raytchev, B. 1991, A\&AS, 89, 77

Volk, K. 1992, ApJS, 80, 347

Volk, K., \& Kwok, S. 1985, A\&A, 153, 79

Whitelock, P. A. 1985, MNRAS, 213, 59

Zhang, C. Y. 1993, ApJ, 410,239

Zhang, C. Y., \& Kwok, S. 1991, A\&A, 250,179 